\title{
Complications of Circumcision
}

\author{
Aaron J. Krill, ${ }^{1}$ Lane S. Palmer, ${ }^{1}$ and Jeffrey S. Palmer ${ }^{2}$ \\ ${ }^{1}$ Division of Pediatric Urology, Cohen Children's Medical Center of New York of the North \\ Shore-Long Island Jewish Health System, Long Island, NY 11042, USA \\ ${ }^{2}$ Pediatric and Adolescent Urology Institute, Beachwood, OH 44122, USA
}

Received 25 June 2011; Accepted 28 September 2011

Academic Editor: Anthony Atala

In the United States, circumcision is a commonly performed procedure. It is a relatively safe procedure with a low overall complication rate. Most complications are minor and can be managed easily. Though uncommon, complications of circumcision do represent a significant percentage of cases seen by pediatric urologists. Often they require surgical correction that results in a significant cost to the health care system. Severe complications are quite rare, but death has been reported as a result in some cases. A thorough and complete preoperative evaluation, focusing on bleeding history and birth history, is imperative. Proper selection of patients based on age and anatomic considerations as well as proper sterile surgical technique are critical to prevent future circumcision-related adverse events.

KEYWORDS: Penis, circumcision, complications, child, micropenis, inconspicuous 


\section{BACKGROUND}

Circumcision is one of the oldest surgical procedures and one of the most commonly performed surgical procedures in practice today $[1,2]$. Descriptions of ritual circumcision span across cultures, and have been described in ancient Egyptian texts as well as the Old Testament. Approximately, 1.1 million neonatal circumcisions were performed in the USA in 2008 alone [3], and the incidence of this procedure appears to be rising. In a large retrospective review of the Nationwide Inpatient Sample, estimated rates of newborn circumcision have risen from $48.3 \%$ nationwide in the years $1988-91$ to $61.1 \%$ of male newborns from 1997-2001. This represents an increase in incidence of approximately $6.8 \%$ per year [1]. This may be due in part to the American Association of Pediatrics modification of its stance in 1989 to neither recommend nor condemn routine neonatal circumcision [4]. Various authors have reported benefits including: prevention of urinary tract infections and pyelonephritis, decreased rates of penile cancer, and decreased HIV transmission [5-8]. However, circumcision, like any surgical procedure, does carry the risk of complications. Complication rates depend on multiple factors, including anatomic abnormalities, medical comorbidities, surgical technique, and patient age. This paper will deal with the most common complications and methods to manage them.

\section{METHODS OF CIRCUMCISION}

Various methods of circumcision are commonly used in the United States today. The choice of circumcision method depends on the physician's level of comfort and training. The most commonly utilized techniques used in the newborn nursery setting are the Gomco clamp, the Mogen clamp, and the Plastibell. While all of these can be used in the operating room, the "free-hand circumcision" using either the sleeve technique or the dorsal-ventral slit technique is most commonly used. Each instrument and technique carries its own benefits and complication risks.

The Gomco clamp is a suture-less technique that utilizes a 4-piece device that protects the glans, provides hemostasis and a platform for resecting the prepuce (Figure 1). The circumcision starts by retracting the foreskin to free the adhesions and allow exposure and inspection of the glans for any abnormalities. The metal bell is placed completely over glans shielding it from damage, followed by placement of the platform over the bell and prepuce caring not to draw the skin up too much (the goal is to maintain the penoscrotal junction), placement of the crimping part which will provide hemostatic compression of the skin after tightening down the screw, excision of the prepuce, disassembly of the apparatus without unsealing the skin edges, and, finally, dressing the wound.

Complications from a Gomco circumcision are mainly related to technical factors. It is important to assure that the metal bell completely covers the glans, otherwise insufficient skin will be removed and accidental incision into the glans is possible. A bell which is too large will result in removal of too much skin. As mentioned, overly aggressive retraction of the skin through the platform can lead to excessive skin removal and subsequent corrective surgery; conversely, insufficient drawing up of the skin will lead to an incomplete circumcision, which may also require corrective surgery. Insufficient tightening of the screw results in inadequate compression of the skin and subsequent bleeding. One may consider keeping the screw tightened for several minutes of hemostatic compression before excising the skin.

The Mogen clamp (Figure 2) is a device used for Jewish ritual circumcisions and commonly used by obstetricians, that also serves to provide hemostasis and a platform for adequate skin removal. After lysis of adhesions and inspection of the glans, the edges of the prepuce are elevated and the V-shaped clamp is placed across the prepuce at the desired location assuring that the glans is positioned below the clamp. The clamp is then tightened to provide hemostasis and the skin is amputated. The complications specific to this techniques include injury to the glans if it is not below the inferior edge of the clamp, and an asymmetric incomplete circumcision due to mal-positioning of the clamp (typically more redundancy ventrally). The possible complications include insufficient or excessive skin removal or asymmetric redundancy and glans amputation all due to improper clamp placement. 


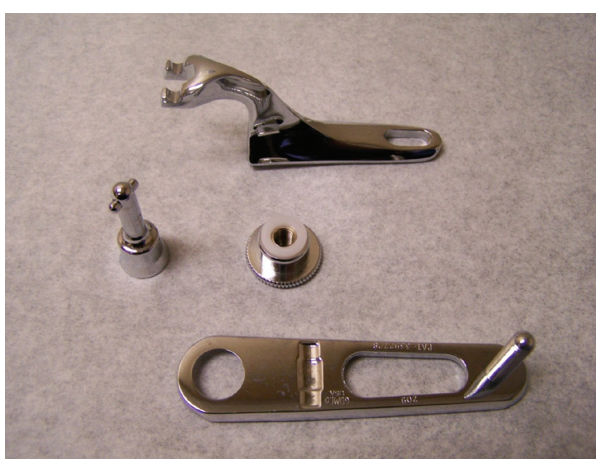

(a)

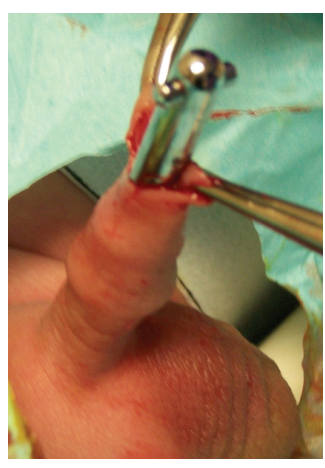

(b)

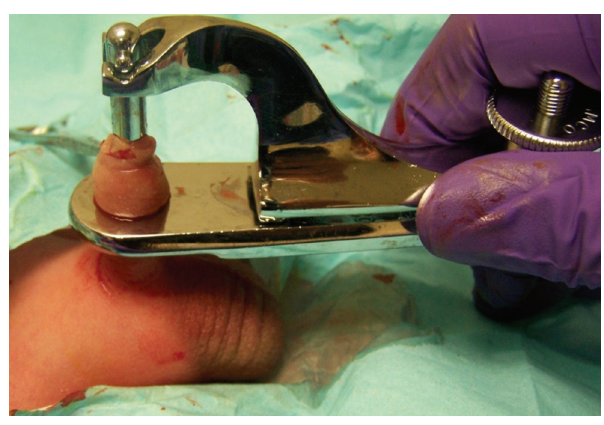

(c)

FIGURE 1: (a) The Gomco clamp consists of 4 pieces: the bell, platform, hooking arm, and screw. These are assembled after placing the bell completely over the glans (b) and the skin drawn through the hole in the platform. Hemostasis is obtained by tightening the screw (c) and the skin excised.

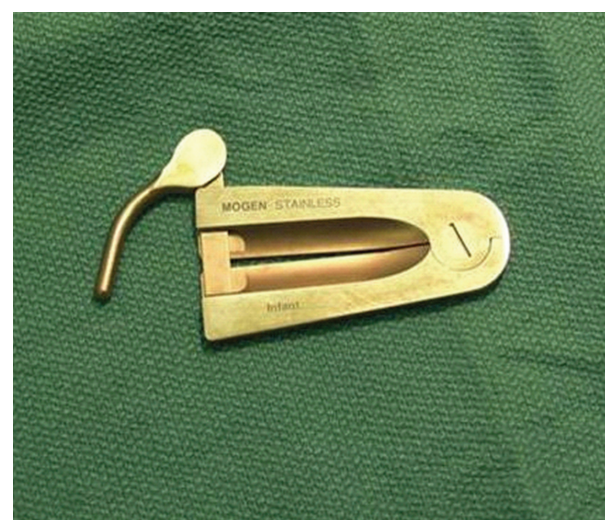

FIGURE 2: The Mogen clamp is used by drawing the skin to be removed into the $V$ and then providing hemostasis followed by amputation.

The Plastibell (Figure 3) technique was developed in the 1950s and is a variation of the Gomco clamp. After lysis of penile adhesions, the plastic bell, similar in appearance to the metal Gomco bell, is placed completely over the glans. Hemostasis is then insured by placing a strangulating suture at the level of the corona and this is all left in place. The skin will slough after a few days and the Plastibell falls off [9]. The associated complications include incomplete circumcision due to inadequate bell placement or slippage of the Plastibell while tying the hemostatic suture, and glans injury or bleeding due to inadequate hemostatic suture placement.

The "free-hand circumcision" is accomplished in the operating room and involves excising the skin as marked, hemostasis using electrocautery and then reapproximation of the skin edges using absorbable sutures or octyl-2-cyanoacrylate (Dermabond ${ }^{\mathrm{TM}}$, Ethicon) [10]. The lines of incision for the "sleeve technique" are circumferential at the same distance from the corona on the inner and outer preputial surfaces (Figure 4). The sleeve of skin is removed and the procedure completed. For the dorsal-ventral slit technique (Figure 5), these incisions are made as their names infer down to the predetermined distance from the coronal edge, and then the incisions are connected leaving the two circumferential free-edges which are then closed after achieving hemostasis. 


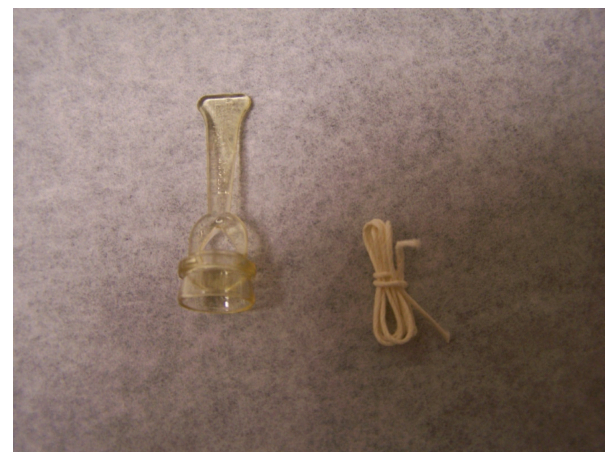

FIGURE 3: The Plastibell is placed over the glans and a suture is secured over the skin. After several days, the skin will slough and the Plastibell falls off.



(a)

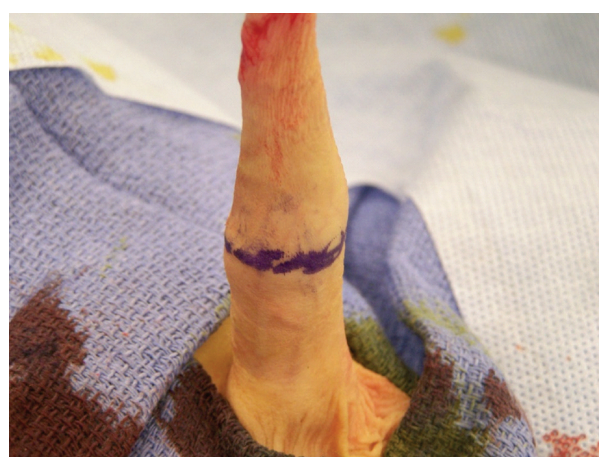

(b)

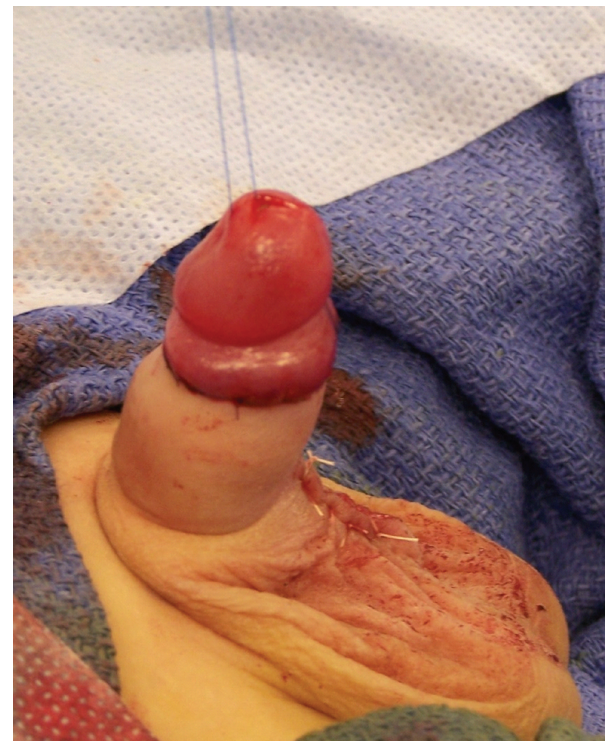

(c)

FIGURE 4: The "sleeve" technique involves incising the inner preputial skin (a) and then the overlying outer preputial skin (b). Sutures are most commonly used to approximate the skin edges (c). 


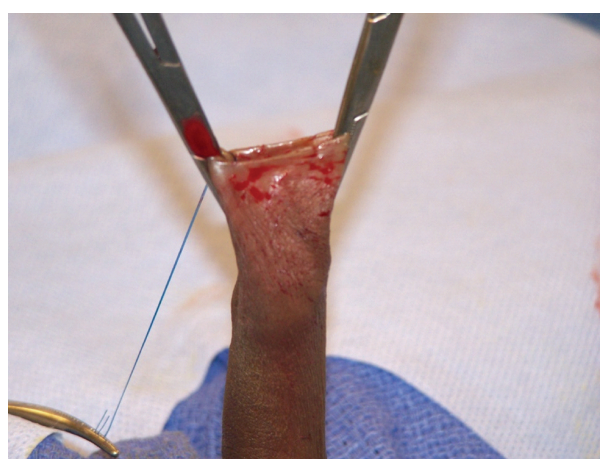

(a)



(b)

FIGURE 5: (a) The dorsal-ventral slit technique of circumcision involves making these incisions and then (b) removal of the skin between them. Sutures are most commonly used to approximate the skin edges.

\section{TIMING OF CIRCUMCISION}

It has been widely reported that circumcision complications occur more frequently with increasing age of the patient. Bleeding becomes more common during the "minipuberty" of infancy that begins at 4 weeks of age and extends to 3 months of age. This is thought to be due to hormonally mediated increase in penile and prepuce size and vascularity [11]. In a recent prospective observation-based study of 583 neonatal circumcisions, Banieghbal reported only two minor bleeding complications requiring sutures. Both occurred in infants aged 3 weeks. Based on use of the Neonatal Infant Pain Scale, he further reported that the ideal timeframe for a "pain free" circumcision is during the first week of life [12]. This is further supported by Horowitz and Gershbein who reported zero complications in 98 infants circumcised with a Gomco clamp in their first month of life versus a 12/32 or a $30 \%$ bleeding complication rate requiring sutures or fulguration in those aged 3-8 months [13].

\section{COMPLICATIONS}

The rate of adverse events varies widely across reports, depending on the definition chosen for a postoperative complication. In a large meta-analysis of prospective and retrospective series, Weiss et al. reported a frequency of adverse events of and for serious adverse events [14]. This can represent a significant cost in terms of utilization resources and healthcare dollars. During a five-year period at the Massachusetts General Hospital, 7.4\% of all visits to a pediatric urologist were for circumcision complications. This translated to an average total cost per patient for redo procedures of \$1,617 and an estimated annual cost of $\$ 137,122$ to the institution [15].

For ease of discussion, adverse events following circumcision can be categorized as either early or late complications. Early complications such as: bleeding, pain, inadequate skin removal, and surgical site infection tend to be minor and quite treatable. However, postcircumcision bleeding in patients with coagulation disorders can be significant and sometimes even fatal. Other serious early complications include chordee, iatrogenic hypospadias, glanular necrosis, and glanular amputation. The latter, of course, requires prompt surgical intervention. Late complications include epidermal inclusion cysts, suture sinus tracts, chordee, inadequate skin removal resulting in redundant foreskin, penile adhesions, phimosis, buried penis, urethrocutaneous fistulae, meatitis, and meatal stenosis. These are commonly treated in an outpatient setting. Most of the aforementioned conditions are avoidable giving attention to detail and proper technique. Mayer et al. found that some subtle anatomic variations are significantly associated with late circumcision complications, including penoscrotal webbing, suprapubic fat pads, and prematurity [16]. 


\subsection{Death}

Fortunately, death from neonatal circumcision is fortunately an extremely rare occurrence. King reported a period when 500,000 consecutive circumcisions were performed in New York city without a single fatality [17]. However, a case of a misplaced Plastibell ring which caused complete meatal obstruction resulted in acute venous stasis and subsequent death from sepsis reported by the Ontario Pediatric Death Review Committee in 2007. In this situation, prompt recognition of the obstruction is critical and primary management should be immediate removal of the Plastibell ring and catheter placement [18]. There are other reports in the international literature that describe mortalities from tetanus as a result of circumcisions performed under nonsterile conditions. Bennett et al. reported that topical antibiotics could decrease this risk of neonatal tetanus 4-fold [19].

\subsection{Bleeding}

Bleeding is the most common complication of circumcision, with an incidence of $1 \%$ in a large retrospective review [20]. Bleeding may occur along the skin edges between sutures or from a discrete blood vessel, most commonly at the frenulum. Meticulous attention to hemostasis during an open procedure and adequate time for skin edge compression during newborn circumcisions should prevent the majority of cases although dislodging of a clot or cautery eschar can occur. The majority of postcircumcision bleeding can be controlled with application of direct pressure or careful application of silver nitrate. Rarely is wound exploration and suturing necessary. A hematologic workup is warranted only in patients who persistently bleed, or bleed significantly. In a retrospective review of the Mayo Clinic Pediatric Hemophilia database, 48 patients with a range of coagulopathies were circumcised. 21 patients had known coagulation disorders, while the remaining 27 patients were diagnosed after prolonged bleeding from their circumcision. There were 11 bleeding complications, three of which were severe and required transfusion of RBC's for severe anemia despite preoperative factor replacement [21]. In those patients with hemophilia who must undergo circumcision, preoperative and perioperative factor replacement is a definite requirement. Fibrin glue has also been shown to decrease the amount of recombinant factor replacement needed (and the cost of treatment, as well) without significantly altering bleeding complications [22].

\subsection{Infection}

Due to the superb dual blood supply of the penis, wound infection occurs infrequently. In a series of 5,521 circumcisions comparing the Plastibell technique to the Gomco clamp, Gee and Ansell reported only 23 (0.4\%) infections. Of those, the Plastibell group had significantly more infections, 19 versus four $(P<$ 0.005) [20]. All responded to a combination of topical treatment and oral antibiotic therapy. Causative organisms are usually skin flora, but due to the uniquely dirty environment of the diaper, colonic flora has also been reported. Most infections can be prevented with proper patient preparation, glove wearing and good local wound care including cleaning the penis, and application of antibiotic ointment with diaper changes [2].

Severe infections following Plastibell circumcision, including necrotizing fasciitis, have been reported as well. Several authors describe presenting signs and symptoms as erythema, induration, pain out of proportion to physical findings, coupled with tachycardia, leucocytosis, or bandemia. As in adults, this is usually a polymicrobial infection. Empiric broad spectrum antibiotics to cover Gram-negative, Grampositive, and anaerobic organisms are essential. A suggested regimen is an aminoglycoside, nafcillin, or vancomycin and clindamycin. Prompt surgical evaluation and aggressive debridement of necrotic tissue is required [23].

\subsection{Loss of Skin/Wound Dehiscence}

Wound dehiscence and degloving injuries of the shaft are possible following using any of the techniques described above for neonatal circumcision. De-gloving injuries result from excess skin being drawn up into 
the clamp and then amputated. While, less likely, improper determination of the amount of skin to remove during free-hand circumcision may occur. Often these injuries are treated with local wound care and allowed to heal by secondary intention. There are reports of autografting the excised skin with good cosmetic result [24].

\subsection{Trapped/Concealed Penis}

Similarly, a concealed penis can result from overzealous removal of shaft skin coupled with a prominent suprapubic fat pad resulting in healing within the fat pad. Another consequence of this configuration is a secondary phimosis from the progressive closure of the skin over the glans penis. This can be avoided by firmly compressing the fat pad to the abdominal wall to best determine how much skin should be removed [16]. In addition, the suprapubic fat should be compressed regularly after the procedure to allow the penis to be protrude. Corrective surgery may be necessary if there is absence or significant laxity of the penoscrotal angle or penoscrotal webbing that precludes adequate protrusion of the penis.

\subsection{Redundant Foreskin/Circumcision Revision (Figure 6)}

Inadequate circumcision, or excess foreskin, is a fairly common indication for referral to a pediatric urologist. In a retrospective review of 476 late circumcision complications treated at Massachusetts General Hospital, $40 \%$ were for inadequate circumcision and another $5 \%$ were for phimosis requiring revision. The criteria for treatment are purely subjective and mainly cosmetic excepting those with phimosis, of course [15]. Repair is scheduled electively under general anesthesia and is best accomplished using the "free-hand" skin sleeve technique removing the redundant skin.

\subsection{Preputial Adhesions/Skin Bridges (Figure 7)}

Preputial adhesions often result from either inadequate lysis of natural adhesions prior to circumcision or from distal migration of the skin from a prominent suprapubic fat pad. Williams et al. report that $63 \%$ of patients presenting for circumcision revision were found to have prominent suprapubic fat pads [25]. The majority of adhesions should lyse spontaneously as the penis grows, suprapubic fat recedes, and erections become more frequent and firmer. Otherwise, lysis of preputial adhesions can be performed in the office by gently pushing away the adhesions from the glans after administering a topical anesthetic cream such as EMLA. Recurrence of these adhesions can be limited by regular compression of the suprapubic area to make the penis protrude and placement of petroleum jelly or any other such ointment. During healing, the circumferential incision can adhere to the glans and in some cases heal into an epithelialized skin bridge [2]. If thin and transparent, they can be divided in the office. However, extensive adhesions and thick skin bridges require surgical intervention. These can be divided sharply after a period of compression with a hemostat. Skin bridges accounted for nearly $30 \%$ of the late complications reported by the Massachusetts General Hospital group [15].

\subsection{Meatitis/Meatal Stenosis (Figure 8)}

In the absence of the prepuce, the erythema of the meatus commonly occurs after circumcision as a result of irritation. Meatitis is commonly a self-limited problem but can be treated by application of an antibiotic ointment and keeping the area dry. Meatal stenosis has been postulated to be a result of ligation of the frenular artery or from ammoniacal meatitis [2] and account for $26 \%$ of the late complications [15]. While the meatus may appear small, it can open adequately during voiding; this explains why most children with meatal stenosis do not present until after toilet training. Surgical treatment is indicated in the presence of symptoms such as deflection of the urine stream, dripping, dysuria, or urgency frequency from inadequate 


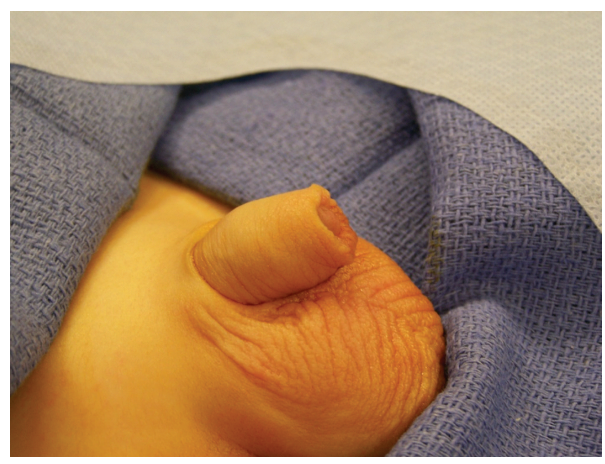

(a)

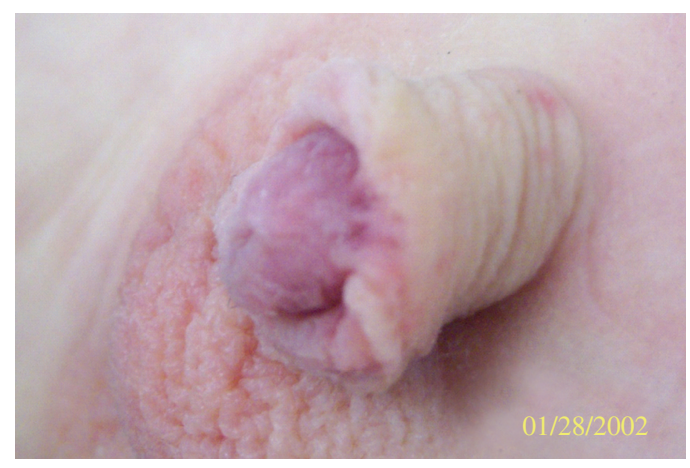

(b)

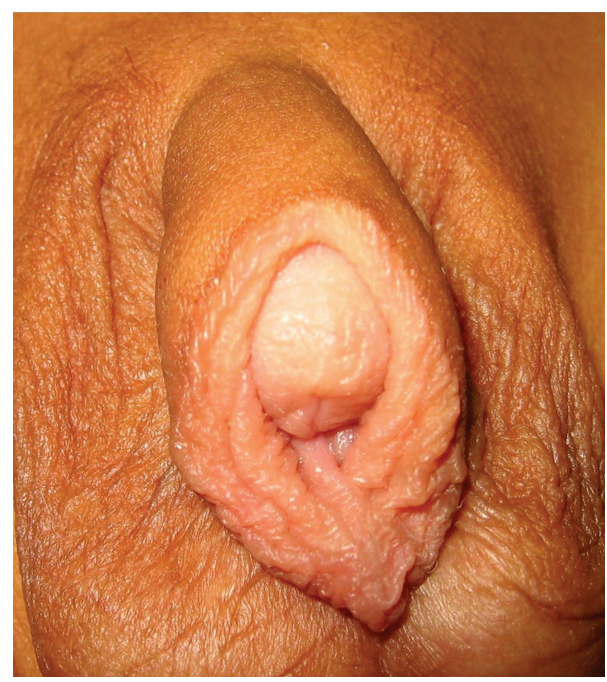

(c)

FIGURE 6: Examples of redundant skin following circumcision (a,b,c).

emptying. The treatment is meatotomy or meatoplasty, which can be accomplished under local anesthesia in the office or in the operating room.

\subsection{Urethrocutaneous Fistula}

Urethrocutaneous fistula is a rare complication, but nonetheless has been reported after both Plastibell and Gomco circumcisions [9]. Fistulae may present as an obvious fistulous tract or as a split urine stream. Often this is a result of compression necrosis from a retained Plastibell ring or a direct injury from incorrect placement of the Gomco clamp. Injury to the urethra during any ventral dissection can occur during a "freehand" circumcision. Delayed flap repair can be done electively after the child's penis has grown enough for good tissue handling.

\subsection{Glanular Necrosis/Glanular Amputation}

Necrosis of the glans can occur as a result of cautery injury during a Gomco circumcision or from distal migration of an incorrectly sized Plastibell ring [9, 20]. Management of glans necrosis depends on its severity. Mild cases can be managed with local wound care and topical antibiotic ointment and allowing 


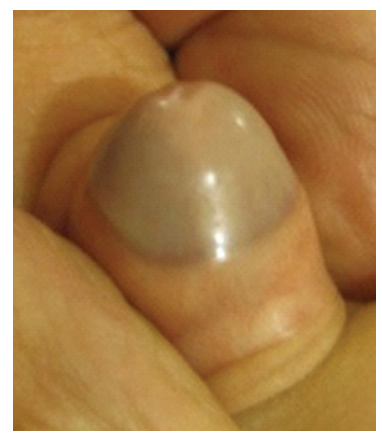

(a)

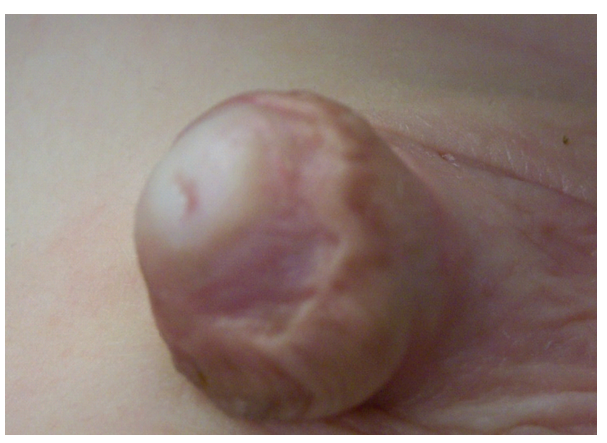

(b)

FIGURE 7: Penile adhesions (a) between the shaft skin and the glans can be manually separated while penile skin bridges (b) cannot be manually separated and need to be excised-skin bridge.

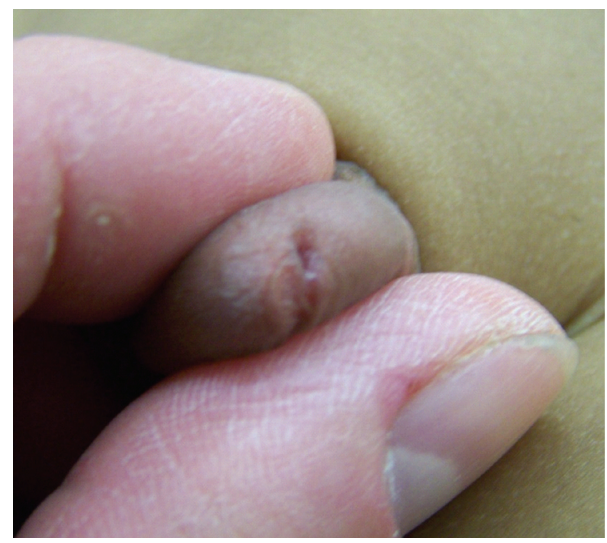

FIGURE 8: Meatal stenosis in a 3 year old circumcised male who presented with a narrow stream.

the necrotic skin to slough. Some authors report management of severe cases with suprapubic diversion and delayed urethroplasty [9]. There are rare case reports of complete necrosis of the glans and phallus in which gender reassignment was performed after multiple attempts at staged repair [20]. For this reason, the use of electrocautery is contraindicated in clamp circumcision.

Amputation of the glans occurs extremely rarely, but is a devastating complication of Mogen clamp circumcision. The Mogen clamp or shield seems uniquely susceptible to this particular injury given the surgeon's inability to directly visualize the glans prior to incising the foreskin. Sherman et al. report seven glanular reconstructions after traumatic amputations. The authors reported that minimal debridement and recovery of the amputated tissue were critical to the repair. Simple primary reanastomosis of glanular tissue was possible in 6 of 7 patients, the last patient required urethral reconstruction [26]. The patient's own tissue can remain viable up to 8 hours and used successfully for repair if adequately preserved by wrapping the tissue in moist saline gauze placed in a plastic bag and transported on ice [26].

\subsection{Hypospadias}

Isolated cases of iatrogenic hypospadias have been reported after the surgeon performed a ventral rather than a dorsal slit prior to initiation of circumcision [27]. It is important that the proper plane be entered for the initial lysis of adhesions so that the meatus is not inadvertently entered and then damaged.

While not technically a complication, failure to recognize a hypospadias prior to circumcision may be problematic if there is insufficient skin for subsequent repair. While most cases of hypospadias 
are associated with a dorsally hooded prepuce, the megameatus with intact prepuce variant will have a configuration as described in its name. Thorough physical examination is imperative prior to circumcision regardless of the method employed.

\section{REFERENCES}

[1] C. P. Nelson, R. Dunn, J. Wan, and J. T. Wei, "The increasing incidence of newborn circumcision: data from the nationwide inpatient sample," Journal of Urology, vol. 173, no. 3, pp. 978-981, 2005.

[2] J. C. Hutcheson, "Male neonatal circumcision: indications, controversies and complications," Urologic Clinics of North America, vol. 31, no. 3, pp. 461-467, 2004.

[3] US Dept of Health and Human Services, http://www.ahrq.gov/.

[4] C. M. Lannon, A. G. D. Bailey, A. R. Fleischman et al., "Circumcision policy statement. American Academy of Pediatrics. Task Force on Circumcision,” Pediatrics, vol. 103, no. 3, pp. 686-693, 1999.

[5] H. G. Rushton and M. Majd, "Pyelonephritis in male infants: how important is the foreskin?" Journal of Urology, vol. 148, no. 2, pp. 733-736, 1992.

[6] R. H. Gray, G. Kigozi, D. Serwadda et al., "Male circumcision for HIV prevention in men in Rakai, Uganda: a randomised trial," The Lancet, vol. 369, no. 9562, pp. 657-666, 2007.

[7] R. C. Bailey, S. Moses, C. B. Parker et al., "Male circumcision for HIV prevention in young men in Kisumu, Kenya: a randomised controlled trial," The Lancet, vol. 369, no. 9562, pp. 643-656, 2007.

[8] B. Auvert, D. Taljaard, E. Lagarde, J. Sobngwi-Tambekou, R. Sitta, and A. Puren, "Randomized, controlled intervention trial of male circumcision for reduction of HIV infection risk: the ANRS 1265 trial," PLoS Medicine, vol. 2, no. 11, article e298, 2005.

[9] C. O. Bode, S. Ikhisemojie, and A. O. Ademuyiwa, "Penile injuries from proximal migration of the Plastibell circumcision ring," Journal of Pediatric Urology, vol. 6, no. 1, pp. 23-27, 2010.

[10] J. M. Elmore, E. A. Smith, and A. J. Kirsch, "Sutureless circumcision using 2-octyl cyanoacrylate (Dermabond): appraisal after 18-month experience," Urology, vol. 70, no. 4, pp. 803-806, 2007.

[11] D. A. Damassa and J. M. Cates, "Sex hormone-binding globulin and male sexual development," Neuroscience and Biobehavioral Reviews, vol. 19, no. 2, pp. 165-175, 1995.

[12] B. Banieghbal, "Optimal time for neonatal circumcision: an observation-based study," Journal of Pediatric Urology, vol. 5, no. 5, pp. 359-362, 2009.

[13] M. Horowitz and A. B. Gershbein, "Gomco circumcision: when is it safe?" Journal of Pediatric Surgery, vol. 36, no. 7, pp. 1047-1049, 2001.

[14] H. A. Weiss, N. Larke, D. Halperin, and I. Schenker, "Complications of circumcision in male neonates, infants and children: a systematic review," BMC Urology, vol. 10, article 2, 2010.

[15] R. V. Pieretti, A. M. Goldstein, and R. Pieretti-Vanmarcke, "Late complications of newborn circumcision: a common and avoidable problem," Pediatric Surgery International, vol. 26, no. 5, pp. 515-518, 2010.

[16] E. Mayer, D. J. Caruso, M. Ankem, M. C. Fisher, K. B. Cummings, and J. G. Barone, "Anatomic variants associated with newborn circumcision complications," The Canadian Journal of Urology, vol. 10, no. 5, pp. 2013-2016, 2003.

[17] L. R. King, "Neonatal circumcision in the United States in 1982," Journal of Urology, vol. 128, no. 5, pp. 11351136, 1982.

[18] J. Cairns, "Circumcision: a minor procedure?" Paediatrics and Child Health, vol. 12, no. 4, pp. 311-312, 2007.

[19] J. Bennett, C. Breen, H. Traverso, S. Bano Agha, J. Macia, and J. Boring, "Circumcision and neonatal tetanus: disclosure of risk and its reduction by topical antibiotics," International Journal of Epidemiology, vol. 28, no. 2, pp. 263-266, 1999.

[20] W. F. Gee and J. S. Ansell, "Neonatal circumcision: a ten year overview: with comparison of the Gomco clamp and the Plastibell device," Pediatrics, vol. 58, no. 6, pp. 824-827, 1976.

[21] V. Rodriguez, R. Titapiwatanakun, C. Moir, K. A. Schmidt, and R. K. Pruthi, "To circumcise or not to circumcise? Circumcision in patients with bleeding disorders," Haemophilia, vol. 16, no. 2, pp. 272-276, 2010.

[22] A. Avanoglu, A. Çelik, I. Ulman et al., "Safer circumcision in patients with haemophilia: the use of fibrin glue for local haemostasis," BJU International, vol. 83, no. 1, pp. 91-94, 1999. 
[23] D. P. Bliss, P. J. Healey, and J. H. T. Waldhausen, "Necrotizing fasciitis after Plastibell circumcision," Journal of Pediatrics, vol. 131, no. 3, pp. 459-462, 1997.

[24] H. I. Patel, K. P. Moriarty, P. A. Brisson, and N. R. Feins, "Genitourinary injuries in the newborn," Journal of Pediatric Surgery, vol. 36, no. 1, pp. 235-239, 2001.

[25] C. P. Williams, B. G. Richardson, and T. P. Bukowski, "Importance of identifying the inconspicuous penis: prevention of circumcision complications," Urology, vol. 56, no. 1, pp. 140-143, 2000.

[26] J. Sherman, J. G. Borer, M. Horowitz, and K. I. Glassberg, "Circumcision: successful glanular reconstruction and survival following traumatic amputation," Journal of Urology, vol. 156, no. 2, pp. 842-844, 1996.

[27] S. D. Niku, J. A. Stock, and G. W. Kaplan, "Neonatal circumcision," Urologic Clinics of North America, vol. 22, no. 1, pp. 57-65, 1995.

\section{This article should be cited as follows:}

Aaron J. Krill, Lane S. Palmer, and Jeffrey S. Palmer, "Complications of Circumcision," TheScientificWorldJOURNAL, vol. 11, pp. 2458-2468, 2011. 


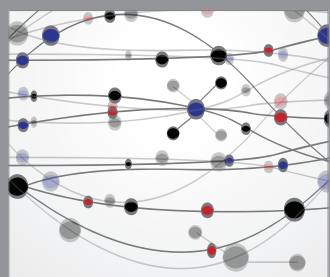

The Scientific World Journal
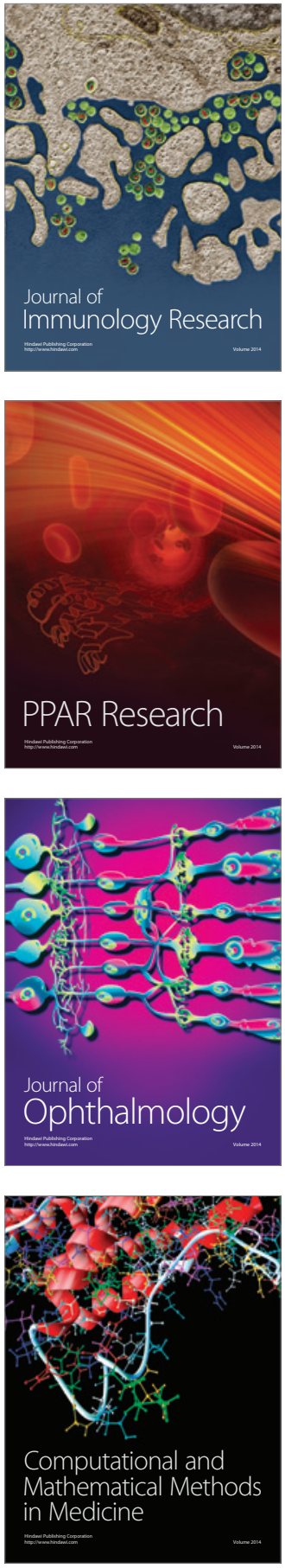

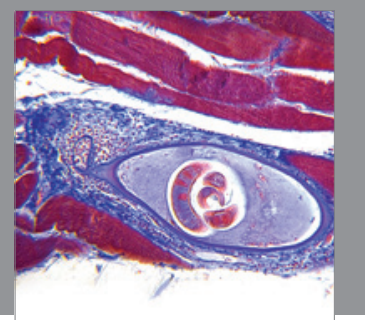

Gastroenterology

Research and Practice
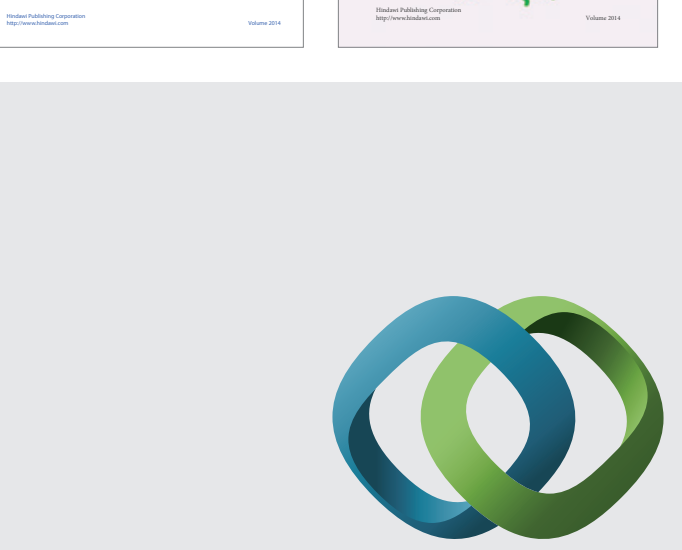

\section{Hindawi}

Submit your manuscripts at

http://www.hindawi.com
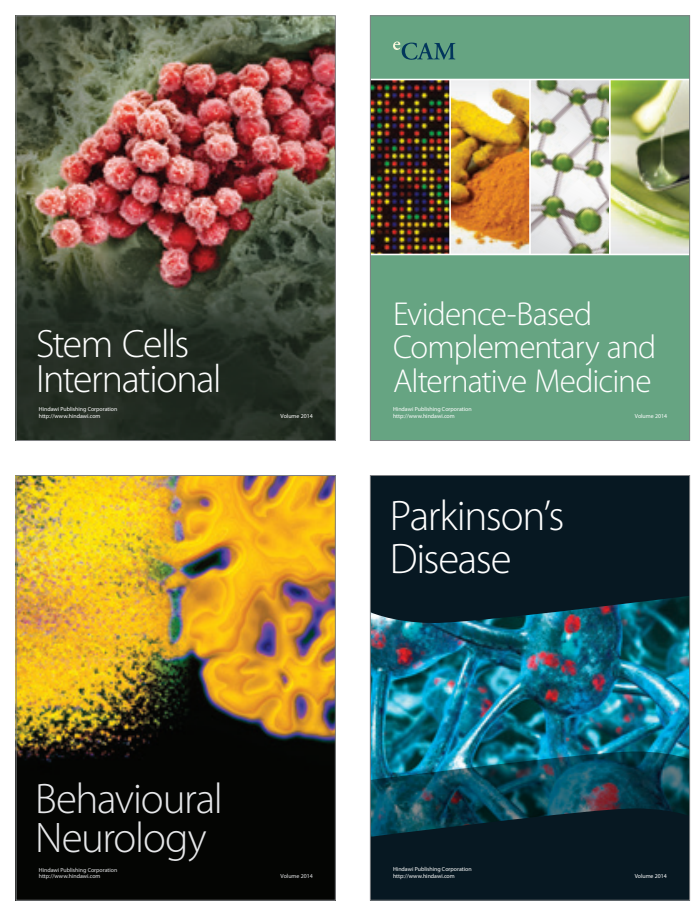

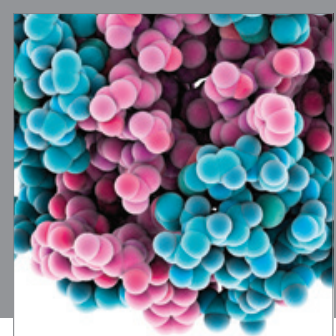

Journal of
Diabetes Research

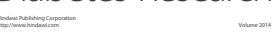



Disease Markers
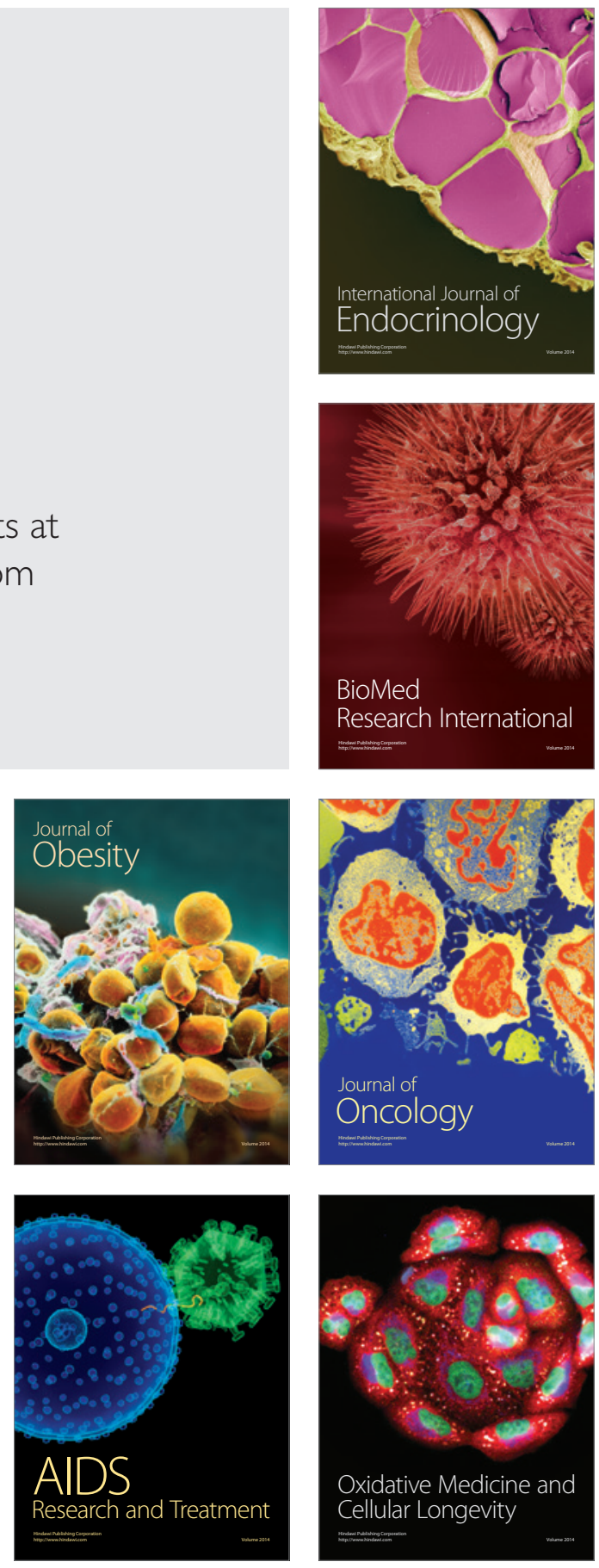